\title{
A NUMERICAL MODEL FOR BUBBLE SIZE DISTRIBUTION IN TURBULENT GAS-LIQUID DISPERSION
}

\author{
M. A. K. Azad and Sultana R. Syeda* \\ Department of Chemical Engineering \\ Bangladesh University of Engineering and Technology, Dhaka 1000, Bangladesh
}

\begin{abstract}
In present study a numerical model for bubble size distribution is developed that considers both breakage and coalescence in turbulent gas liquid dispersion. Two-step mechanisms are considered for both breakage and coalescence of bubbles. The bubble breakage is structured as the product of the bubble-eddy collision frequency and breakage efficiency in gas-liquid dispersions. The coalescence function considers the product of bubble-bubble collision frequency and coalescence efficiency. The model overcomes several limitations observed in previous efforts such as empirical parameters, narrow range of operating conditions, and narrow range of geometries. Favorable agreement is found between the predicted bubble size distribution and the experimental data reported in the literature. The percentage of error obtained for the average bubble size was found within $\pm 17 \%$.
\end{abstract}

\section{Introduction}

Bubble columns are employed in many mass transfer processes. They are used in a variety of industrial applications ranging from stripping and absorption columns to three phase slurry beds and activated sludge ponds. Bubble columns have broad application as reactors and separation units in the chemical, mining, pharmaceutical and biochemical industries. Bubble columns are also used as aerobic biochemical reactors in fermentation systems.

There is considerable interest in developing means of predicting bubble-size distributions in turbulent two-phase dispersions. An understanding of the physical mechanisms determining bubble size is crucial to any detailed theory of the transfer of heat, mass and momentum between phases, and is also necessary for the framing of the design of reduced-scale laboratory models to simulate bubble and droplet flows in industrial plant.

Bubble size distribution in a vessel is not constant, but may change due to bubble-bubble interactions that can lead to breakage or coalescence. No broadly applicable model for the determination of these two rates has yet been presented due to both the unsatisfactory understanding of the physical mechanisms that lead to breakage and coalescence and the enormous difficulty in obtaining reliable data, especially for high gas flow rates.

Most of the studies investigating bubble size distribution in turbulent flow, dealt with breakage and coalescence separately. The primary-contributions to the study of Bubble breakup in turbulent flow are those of Kolmogoroff ${ }^{1}$ and Hinze ${ }^{2}$. These authors independently suggested that the maximum size of bubble stable against breakup by the turbulence could be estimated by means of dimensional analysis based on the hypothesis that the key parameter characterizing the structure of turbulence fluctuations is the rate of energy dissipation in the flow. Similar line of argument applied to coalescence, suggests that in a turbulent environment there is a minimum size of bubble stable against coalescence Shinnar ${ }^{3}$. Following the footsteps of Kolmogroff and Hinze a number of studies were carried out that dealt with either breakage or coalescence phenomena separately ${ }^{4,5,6,7,8,9}$.

Prince and Blanch $^{10}$ are among the very few researchers who proposed a phenomenological model for the rates of bubble coalescence and bubble break up in bubble columns. They modeled bubble coalescence by considering bubble collisions due to turbulence, buoyancy and laminar shear and by analyzing the coalescence efficiency of collisions. Bubble break up was estimated in terms of bubble interactions with turbulent eddies. They developed a method to measure the coalescence and breakup events in turbulent systems and used the measurements to validate their model. However, lack of information on several parameters makes the model difficult for application.

Colella et. al. ${ }^{11}$ studied the interfacial mechanisms that dealt with both coalescence and breakage of bubbles. They tried to develop a new methodology to analyze breakage and coalescence phenomena in bubble columns based on the physics of bubble columns and by considering wake and shape effect. Again, several adjustable parameters included in the model, made the model case sensitive.

The present study is aimed at developing a general theory based model to determine equilibrium bubble size distribution in gas liquid dispersion without including any adjustable parameter. The objectives will be fulfilled through the following steps. 
1. Formulation of the turbulent break up and coalescence events.

2. Development of an algorithm where both breakup and coalescence are considered simultaneously towards attainment of equilibrium bubble size distribution.

3. Comparison of the predicted bubble size distribution with experimental data available in literature.

\section{Model Development}

\section{Initial bubble size distribution}

Initial bubbles, i.e., bubbles just after leaving sparger orifices or nozzles are different in size from the bubbles rising through the main part of the column under the operating conditions normally used in practice. The size of the majority of bubbles at equilibrium distribution depends mainly on input bubble size and a balance between the coalescence and breakup rates. Experimental data showed that the initial bubble size was independent of the properties of system such as surface tension, liquid viscosity and liquid and gas densities ${ }^{12}$. It is also reported that the size distribution of such bubbles is log normal distribution $^{13}$. The distribution function $F(x)$ is defined by

$$
F(x)=\int_{0}^{x} f(x) d x
$$

Where $f(x)$ is the probability density function. The probability density function $f(x)$ can be expressed by

$$
f(x)=\frac{1}{x(\sigma) \sqrt{2 \pi}} \exp \left(-\frac{1}{2}\left(\frac{\ln x-m}{\Sigma}\right)^{2}\right)
$$

Where $m$ is the natural logarithm of the geometric mean bubble size $\left(d_{g}\right)$, and $\Sigma$ is the standard deviation. Here geometric mean is assumed equal to the average mean of bubble size. The average bubble size of initial bubbles depends on the orifice diameter $d_{o}$ and the gas velocity through the orifice $u_{0}{ }^{12}$.

$d_{g}=d_{o}\left(1.88\left(u_{o} / \sqrt{g d_{o}}\right)^{1 / 3}\right)$

From dimensional analysis and the experimental data, the following correlation was obtained for the standard deviation of bubble size distribution.

$$
\Sigma=\sqrt{\ln \left(5.60\left(g D^{3} / v_{l}^{2}\right)^{-.08}\left(U_{g} / \sqrt{g D}\right)^{-0.08}\right)}
$$

Bubble concentrations can be obtained from gas holdup and bubble size data. Taking the number of bubbles as equal to the volume of gas divided by the average bubble size one obtains:

$n_{i}=\frac{\phi R_{T}^{2} H_{T}}{\frac{4}{3} \overline{r_{b i}^{3}}} f\left(x_{i}\right)$

Here $f\left(x_{i}\right)=$ the fraction of bubbles with radius $r_{b i}$

For determining total number of bubbles initially present in the system $f\left(x_{i}\right)$ is considered as 1 and average bubble size is determined from the Eq. 3 .

$n_{T}=\frac{\phi R_{T}^{2} H_{T}}{\frac{4}{3} \overline{r_{b i}^{3}}}$

The correlation of Hikita et.al. ${ }^{14}$ allows one to estimate the global gas hold up based on the physical properties of the phase.

$\phi=0.672\left(\frac{\mu_{c} \mu_{d}}{\sigma}\right)^{0.578}\left(\frac{\mu_{c}^{4} g}{\rho_{l} \sigma^{3}}\right)^{-0.131}\left(\frac{\rho_{c}}{\rho_{d}}\right)^{0.062}\left(\frac{\mu_{d}}{\mu_{c}}\right)^{0.107}$

In present study the bubble size distribution within $12.5 \mathrm{~cm}$ from the bottom of the column is considered to have initial bubble size distribution.

\section{Bubble coalescence theory}

Coalescence of two bubbles in turbulent flows occurs in three steps.

1. Collisions of bubbles and trapping of liquid between them.

2. Flattening of bubble surface and drainage of the trapped liquids.

3. Coalescence of two bubbles.

From the first step it is seen that the coalescence rate is intimately connected to the collision rate. The second step is ordinarily the slower and hence determines the overall duration of the coalescence process. Collision occurs due to the following mechanisms:

i) Turbulence (Random motion of bubbles due to turbulence)

ii) Buoyancy (Bubbles of different sizes will have different rise velocities which may lead to collision)

iii) Laminar shear (Bubbles located in a region of relatively high liquid velocity may collide with bubbles in slower section of the velocity field)

It is assumed that collisions from these various mechanisms are cumulative. 


\section{Turbulent collision rate}

Collision takes place by mechanism analogous to particle collisions in an ideal gas. The primary reason for bubble collision is the fluctuating turbulent velocity of the liquid phase. The turbulent motion can be expressed as a function of bubble size, concentration and

$$
\text { velocity }^{15} \quad \theta_{i j}^{T}=n_{i} n_{j} S_{i j}\left(\bar{u}_{t i}^{2}+\bar{u}_{t j}^{2}\right)^{1 / 2}
$$

Where $n_{i}, n_{j}$ are the concentration of bubbles of radius $r_{b i}, r_{b j}$ respectively. $u_{t}$ is the average turbulent fluctuating velocity of the bubble. $S_{i j}$ is the collision cross sectional area of the bubbles, given by

$$
S_{i j}=\frac{\pi}{4}\left(d_{b i}+d_{b j}\right)^{2}
$$

The following assumptions are made to develop the turbulent collision rate.

1. The velocity of bubbles in Eq. 8 is assumed to be the turbulent eddy velocity of the length scale of the bubble.

2. The turbulence is isotropic and that the bubble size lies in the inertial sub range.

This criterion is typically examined in terms of the inverse radius or wave number, which is known as the inertial subrange:

$k_{e}<<k_{b}<<k_{d}$

Where, $\mathrm{k}_{\mathrm{e}}$ is the wave number of the large energy containing eddies; $\mathrm{k}_{\mathrm{b}}$ is the wave number of the corresponding bubble size; $\mathrm{k}_{\mathrm{d}}$ is the wave number of the eddies of viscous dissipation.

Batchelor $^{15}$ defines the wave number for energy dissipation $\left(k_{d}\right)$, equivalent to the inverse of the micro scale of turbulence:

$k_{d}=0.5 \frac{\varepsilon^{1 / 4}}{v^{3 / 4}}$

Where $\varepsilon$ is the energy dissipation per unit mass, $v$ is the kinematic viscosity

An expression for the energy dissipation per unit mass is given by:

$$
\varepsilon=u_{s} \times g
$$

The kinetic energy and mass of the gas phase have been neglected in this formulation.
The size of the energy containing eddies is typically assumed to be equal to the vessel diameter. Thus $k_{e}$ is inverse of vessel diameter. According to Eq.10 the length scale of the bubble is well removed from that of both the energy containing eddies and eddies of viscous dissipation and falls in the inertial subrange.

The turbulent velocity in the inertial subrange of isotropic turbulence is:

$u_{t}=1.04 \varepsilon^{(1 / 3)} d_{b}^{(1 / 3)}$

Where $d_{b}=$ the bubble diameter.

Substitution of this value in to Eq. 8 yields the turbulent collision rate.

$\theta_{i j}^{T}=0.089 \pi n_{i} n_{j}\left(d_{b i}+d_{b j}\right)^{2} \varepsilon^{1 / 3}\left(d_{b i}^{2 / 3}+d_{b j}^{2 / 3}\right)^{1 / 2}$

\section{Buoyancy-driven collision rate}

Collisions may result from the difference in rise velocities of bubbles of different size. The buoyant collision rate $\left(\theta_{i j}{ }^{B}\right)$ is given by Friedlander ${ }^{17}$ :

$\theta_{i j}^{B}=n_{i} n_{j} S_{i j}\left(u_{r i}-u_{r j}\right)$

Where $u_{r}$ is the rise velocity of the bubble and can estimated by the following expression ${ }^{18}$ :

$u_{r}=\left[(2.14 \sigma) /\left(\rho_{l} d_{b}\right)+0.505 g d_{b}\right]^{1 / 2}$

Where $\rho_{l}$ is the liquid density, $\sigma$ is the surface tension. The equation is strictly applicable only to uncontaminated bubbles with mobile gas-liquid interfaces.

\section{Laminar shear collision rate}

The final contribution to the collision rate results from laminar shear in the liquid phase. Collisions occur in this situation as a result of the development of a gross circulation pattern in a bubble column at a sufficiently high gas flow rates. The circulation pattern gives rise to a radial velocity distribution. Because of this, it is possible for bubbles situated in a zone of relatively high liquid velocity to overtake another bubble of the same size and rise velocity.

The functional form of the collision rate due to laminar shear is given by Friedlander ${ }^{16}$ :

$\theta_{i j}^{L S}=\frac{4}{3} n_{i} n_{j}\left(r_{b_{i}}+r_{b_{j}}\right)^{3}\left(\frac{d \bar{U}_{l}}{d R}\right)$

Where $U_{l}=$ the liquid circulation velocity, $\mathrm{R}=$ the radial coordinate of the column 
$\frac{d \bar{U}_{l}}{d R}=$ The average shear rate.

For evaluating the average shear rate, the velocity profile developed by Walters and Blanch ${ }^{17}$ for inviscid fluid is used. The exact velocity profile is a function of the position of the stagnant point, the radial distance at which there is no net upward or downward flow. For inviscid systems, Walter and Blanch state that the transition point occurs at a radial position of approximately $0.7 R_{T}$. The velocity profile for this condition is

$$
U_{l}=U_{l, \max }\left(1-R^{2} /\left(\alpha R_{T}\right)^{2}\right)
$$

Where $\alpha R_{T}=$ the transition point, $U_{l, \text { max }}=$ The velocity in the center of the column.

The mean shear rate is found by averaging the local shear rate over the radial dimension of the column. If we substitute $\gamma(R)$ for the local shear rate we find:

$\bar{\gamma}(R)=\frac{\left|2 \int_{0}^{\alpha R_{T}} \gamma(R) \cdot R d R\right|}{\left(\alpha R_{T}\right)^{2}}+\frac{\left|2 \int_{\alpha R_{T}}^{R_{T}} \gamma(R) \cdot R d R\right|}{R_{T}^{2}\left(1-\alpha^{2}\right)}=5.3 \frac{U_{l, \text { max }}}{R_{T}}$

For turbulent flow the maximum liquid circulation velocity is given by

$$
\begin{aligned}
& U_{l, \max }=\left(\frac{2 Q_{M} g H}{\pi d^{2} F_{L 2}}\right)^{1 / 3} \\
& F_{L 2}=\left(1-\frac{1}{\left(\frac{D^{2}}{d^{2}}-1\right)^{2}}\right) \\
& Q_{M}=Q \frac{P_{2}}{P_{L M}}=Q \frac{P_{2} \ln \frac{P_{1}}{P_{2}}}{\left(P_{1}-P_{2}\right)}
\end{aligned}
$$

Where,

$Q=$ gas rate per orifice, $\mathrm{m}^{3} / \mathrm{s}$.

$Q_{M}=$ Mean gas rate, $\mathrm{m}^{3} / \mathrm{s}$.

$P_{I}=$ Pressure at the sparger, $\mathrm{N} / \mathrm{m}^{2}$.

$P_{2}=$ Absolute Pressure, $\mathrm{N} / \mathrm{m}^{2}$.

$P_{L M}=$ Log mean Pressure, $\mathrm{N} / \mathrm{m}^{2}$.

Substitution of Eq. 21 and Eq. 22 in to Eq.19 gives the laminar shear collision rate.

\section{Collision efficiency}

Collision efficiency is the fraction of bubble collisions that leads to coalescence. The efficiency depends on the contact time between bubbles and the time required for bubbles to coalesce. An expression for the efficiency is given by Coulaloglou and Tavlarides ${ }^{18}$ :

$\lambda_{i j}=\exp \left(-t_{i j} / \tau_{i j}\right)$

Where $t_{i j}=$ The time required for coalescence of bubbles of radius $r_{b i}$ and $r_{b j} ; \tau_{i j}=$ The contact times for the two bubbles. Coalescence times have been successfully modeled in stagnant fluids by examining the time required for the liquid film between bubbles to thin from an initial thickness to a critical value where rupture occurs. An expression for the thinning of liquid film between bubbles of equal size is taken from Oolman and Blanch ${ }^{19}$.

$\frac{-d h}{d t}=\left(\frac{8}{R_{d}^{2} \rho_{l}}\left(\frac{-4 c}{R_{g} T}\left(\frac{d \sigma}{d c}\right)^{2}+h^{2}\left(\frac{2 \sigma}{r_{b}}+\frac{A}{6 \pi h^{3}}\right)\right)\right)^{1 / 2}$

Where $h=$ the film thickness, $R_{d}=$ the radius of the liquid disk between the coalescing bubbles $R_{g}=$ the gas constant, $T=$ the temperature, $A=$ the Hamaker constant $c=$ concentration of a surfactant species. For the distilled water $c=0$.

The coalescence time can be found by integrating of Eq. 24:

$t_{i j}=\left(\frac{r_{i j}^{3} \rho_{l}}{16 \sigma}\right)^{1 / 2} \ln \frac{h_{o}}{h_{f}}$

Where $h_{o}=$ the initial film thickness, $h_{f}=$ the critical thickness where rupture occurs.

$R_{d}$ has been assumed to be bubble radius. This assumption is not strictly accurate; however, it is used for simplicity and because more detailed data is not available. For the case of bubbles of unequal size, $r_{b}$ in Eq. 25 has been replaced by the equivalent radius $\left(r_{i j}\right)$. The equivalent radius is given by Chesters and $\operatorname{Hoffman}^{20}$ as:

$r_{i j}=2\left(\frac{1}{r_{b i}}+\frac{1}{r_{b j}}\right)^{-1}$

The time that bubbles remain in contact is dependent on the bubble size and turbulent intensity. High levels of turbulence increase the probability that an eddy will separate particles, while large particle size provides 
larger contact areas. Levich ${ }^{21}$ provides an estimate of the contact time in turbulent flows:

$$
\tau_{i j}=\frac{r_{b}^{2 / 3}}{\varepsilon^{1 / 3}}
$$

Values for the coalescence and contact time may be substituted in to Eq.23 to determine the efficiency.

In turbulent dispersion a simple criterion for coalescence to occur is $t_{i j}<\tau_{i j}$. In other words unless the intervening film thins down to the critical rupture thickness $h$ in the time available before bubbles are separated again, coalescence doesn't occur. Combining the Eq. 25 and 27 we obtain the result that coalescence is impossible unless $d<d_{\text {min }}$. Where

$d_{\min } \approx 10.55\left(\frac{\left(\sigma / \rho_{l}\right)^{1 / 2}}{\varepsilon^{1 / 3} \ln \frac{h_{0}}{h_{f}}}\right)^{6 / 5}$

Now it can be said that bubbles whose diameter exceeds $d_{\min }$ are much less likely to coalesce than smaller bubbles in the dispersion. Similarly bubbles slightly below the threshold size will not coalesce as easily as very small bubbles. Thus the equation gives a rough estimate of the size of the smallest bubble stable against coalescence

\section{Total coalescence rate}

The coalescence rate of bubbles of radii $r_{b i}$ and $r_{b j}$ is given by the total collision frequency multiplied by the efficiency.

$$
\Gamma_{i j}=\left(\theta_{i j}^{T}+\theta_{i j}^{B}+\theta_{i j}^{L S}\right) \exp \left(\frac{-t_{i j}}{\tau_{i j}}\right)
$$

The overall coalescence rate is then given by:

$$
\Gamma_{T}=\frac{1}{2} \sum_{i} \sum_{j}\left(\left(\theta_{i j}^{T}+\theta_{i j}^{B}+\theta_{i j}^{L S}\right) \exp \left(\frac{-t_{i j}}{\tau_{i j}}\right)\right)
$$

The factor $1 / 2$ is included to avoid counting coalescence events between bubble pair twice.

\section{Bubble breakup theory}

In order to develop the breakage model, the following simplifications are made.
1. The turbulence is assumed to be isotropic.

2. Only the binary breakage of fluid particles is considered.

3. The occurrence of breakup is determined by the energy level of the arriving eddy only.

4. Only eddies of length scale smaller than or equal to the particle diameter can induce particle oscillations.

\section{Collision of bubbles with turbulent eddies}

To obtain an expression for the break-up rate of bubbles, the turbulent collision rate of bubbles with eddies of the appropriate size is considered. The collision rate is given $\mathrm{by}^{22}$ :

$\theta_{i e}=n_{i} n_{e} S_{i e}\left(\overline{u_{t i}^{2}}+\overline{u_{t e}^{2}}\right)^{1 / 2}$

This Eq. is analogous to Eq.8 except that the diameter, concentration and velocity of one bubble are replaced by those of the eddy. To employ this equation, the number of eddies of a particular size must be determined. We assume that the turbulence is isotropic and that the eddy size of interest lies in the inertial subrange.

Batchelor $^{16}$ gives the spectral energy density in the inertial subrange of the energy spectrum as:

$E(k)=1.7 \varepsilon^{2 / 3} k^{-5 / 3}$

Where, $k$ is the eddy wave number $\left(k=1 / r_{e}, r_{e}\right.$ being the radius of the eddy), $\varepsilon$ gives the energy dissipation per unit mass and $E(k)$ is the energy per unit mass and per interval of wave number.

According to Azbel ${ }^{23}, E(k)$ can be written as

$E(k)=N(k) e$

Where, $N(k)$ denotes the number of eddies per unit mass of the liquid and per unit interval of wave numbers. Multiplication by the liquid density yields the eddy concentration.

The energy of a single eddy is given by:

$e=\frac{1}{2} m u_{e}^{2}=\frac{2}{3} \pi \rho_{f} r_{e}^{3} u_{e}^{2}$

In Eq. 34, $r_{e}, u_{e}$, and $\mathrm{m}$ denote the eddy radius, velocity and mass respectively and $\rho_{f}$ denotes the fluid density. According to Kolmogorov's for the inertial subrange of the energy spectrum, the eddy velocity is given as:

$u_{e}=2.86\left(\frac{\varepsilon}{k}\right)^{1 / 3}$ 
Eq.32 to 35 are used to determine the number and velocity of eddies. It must be noted that Eq. 33 is only valid for the inertial subrange. The number of eddies predicted by the equation becomes infinitely large as the eddy size approaches zero. Therefore the expression may not be useful to very small eddies which lie in the viscous dissipation range. In practice, therefore, it is necessary to consider an arbitrary size, below which an eddy will not cause breakage. For the present study, this value is set at eddies smaller than $20 \%$ of the bubble size. Such eddies are unlikely to contribute significantly to the overall break-up rate since they posses only $0.5 \%$ of the kinetic energy associated with an eddy equivalent to the bubble in size.

\section{Break-up efficiency}

Only a certain number of bubble-eddy collisions are likely to result in bubble break-up. The criterion of break-up relates the energy of the eddy to the surface tension forces of the bubble. The balance of disruptive and cohesive forces is generally expressed in terms of the dimensionless Weber number.

$$
W_{e}=\frac{u^{2} d_{b} \rho_{l}}{\sigma}
$$

A critical Weber number will exist at the point where cohesive and disruptive forces balance, resulting in a maximum stable size. An expression for the maximum stable bubble size in turbulent gas-liquid flows provided by Kolmogoroff ${ }^{1}$ and Hinze $^{2}$ :

$$
d_{\max }=1.12 \frac{\sigma^{0.6}}{\varepsilon^{0.4} \rho^{0.6}}\left(\frac{\mu_{c}}{\mu_{d}}\right)^{0.1}
$$

Where $d_{\max }$ is the maximum stable bubble size, $V_{l}$ is the total volume of liquid, and $\mu_{c}, \mu_{d}$ are the viscosities of the continuous and dispersed liquid phase, respectively. All bubbles above the $d_{\max }$ will undergo the breakage process provided that the bubbles remain in the turbulent field for sufficient period of time.

From this expression and Eq. 36 and 13, one may obtain a critical Weber number of 2.3 for air bubbles in water. This is translated in to a critical eddy velocity $\left(u_{c i}\right)$ for break-up of a bubble of radius $r_{b i}$

$u_{c i}=1.52\left(\sigma /\left(d_{b i} \rho_{l}\right)\right)^{1 / 2}$

It is necessary to determine which eddies have velocities that exceed this value. To do so, an energy distribution function is required. Angelidou et al. ${ }^{24}$ provide such an expression for a random distribution of energy:

$x\left(E_{e}\right)=\frac{1}{E_{e}} \exp \left(\frac{-E_{e}}{\overline{E_{e}}}\right)$

Where $x(E)=$ Energy distribution function, $E_{e}=$ the kinetic energy of the eddy.

Taking the energy of the eddy as proportional to the square of the velocity yields a function of the following form for the fraction of eddies with sufficient energy to cause rupture ${ }^{18}$

$F(u)=\exp \left(-\left(\frac{u_{c i}^{2}}{u_{t e}^{2}}\right)\right)$

Where $F(u)=$ the fraction of eddies with sufficient energy to cause rupture and $u_{t e}=$ the turbulent velocity of an eddy of radius $r_{e}$.

\section{Total break-up rate}

The break-up rate for a bubble of radius $r_{b i}$ is thus given by:

$\beta_{i}=\sum_{e}\left(\theta_{i e} \exp \left(-\left(u_{c i}^{2} / u_{t e}^{2}\right)\right)\right)$

Here the summation incorporates the contribution to breakup from eddies of various sizes. The total break-up rate for all bubbles there fore is;

$\beta_{T}=\sum_{i} \sum_{e} \theta_{i e} \exp \left(\frac{-u_{c i}^{2}}{u_{t e}^{2}}\right)$

\section{Model Development}

\section{Algorithm of the model}

In present study a simulation program is used to determine the bubble sizes. Initial bubble size distribution is estimated for a particular superficial gas velocity. These initial bubbles are used to initialize the simulation. The population of bubbles is divided into discrete size categories. The bubbles are first checked for the inertial sub range. If bubbles lie within the sub range they are sent either to coalescence or to breakup depending on their size. If the bubbles are larger than $d_{\max }$ they are sent to break-up; if they are smaller than $d_{\min }$ they are sent to coalescence; and if they lie between $d_{\max }$ and $d_{\min }$ they are not subject to further coalescence or breakup. The resulting size distribution is recorded after a number of coalescence and break-up 
events. The procedure is continued until an equilibrium average bubble size is achieved, i.e. further iterations produce no change in the average bubble size or bubble size distributions. The algorithm to determine the equilibrium bubble size distribution by using the

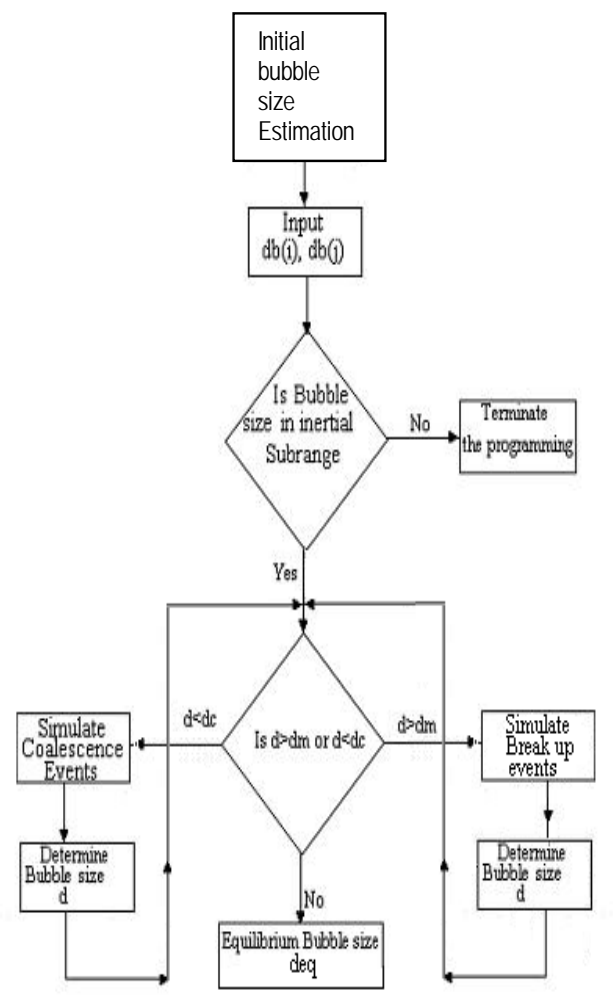

proposed model is shown in Figure 1.

Fig 1. Algorithm for equilibrium bubble size determination

\section{Results and Discussion}

The bubble size distribution determined by the model is compared with the experimental data obtained from bubble columns reported by Collela et. al. ${ }^{11}$ and Prince and Blanch ${ }^{10}$.

The model is first executed with the bubble column used by Colella et al. ${ }^{11}$. The bubble column is made of Plexiglass with a diameter equal to $15.24 \mathrm{~cm}$ and a height of $109 \mathrm{~cm}$.

Bubbles were generated by a perforated plate with holes of $0.1 \mathrm{~cm}$ diameter, arranged in an equilateral triangle pattern with a pitch equal to $1 \mathrm{~cm}$. The distribution is measured for the gas superficial velocity of $0.59 \mathrm{~cm} / \mathrm{s}$ and $1.13 \mathrm{~cm} / \mathrm{s}$. For both velocities the column is operated in homogeneous regime.

Initial bubble size distribution is obtained from the equations 1 to 7 . Figure 2 shows the initial size distribution of the bubble column at gas velocities 0.59 $\mathrm{cm} / \mathrm{s}$ and $1.13 \mathrm{~cm} / \mathrm{s}$.

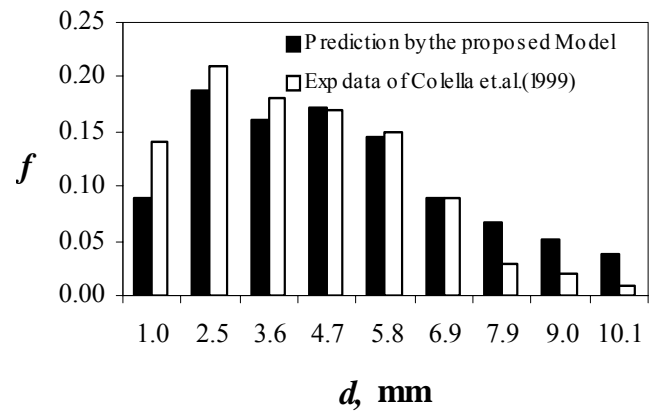

Fig 2. Estimated initial bubble size distribution for the bubble column used by Collela et.al. (1999).

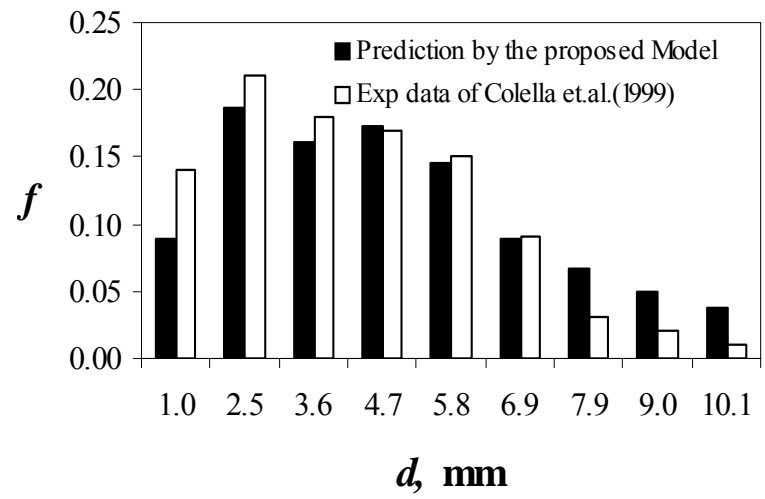

Fig 3. Comparison of the model with the experimental data of Collela et.al. (1999) for velocity $0.59 \mathrm{~cm} / \mathrm{s}$.

The equilibrium size distribution measured at a distance $57.5 \mathrm{~cm}$ from the sparger by Collela et.al. ${ }^{11}$ is compared with the prediction by the proposed model. The result is shown in Figures 3 and 4 for velocities $0.59 \mathrm{~cm} / \mathrm{s}$ and $1.13 \mathrm{~cm} / \mathrm{s}$, respectively. The model is further executed with the bubble column used by Prince and Blanch ${ }^{10}$.

The bubble column consisted of a $27 \mathrm{~cm}$ diameter plexiglass cylinder with a liquid depth of $2 \mathrm{~m}$. The sparger was made up of two $20 \mathrm{~cm}$ long curved stainless steel tubes, each having six orifices of $2 \mathrm{~mm}$ diameter along the upper edge. The two tubes were oriented to maximize radial mixing of the gas streams in the lower section of the bubble column. The distribution was measured at a gas superficial velocity $5.2 \mathrm{~cm} / \mathrm{s}$. For this velocity the column is operated in heterogeneous regime, where turbulent collision and laminar shear collision become dominant. 


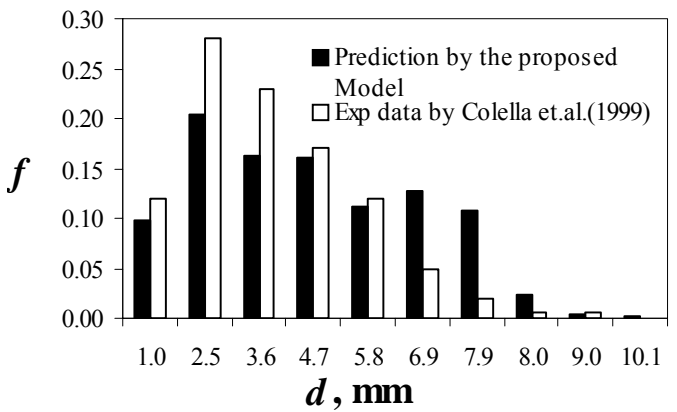

Fig 4. Comparison of the model with the experimental data of Collela et.al. (1999) for velocity $1.13 \mathrm{~cm} / \mathrm{s}$.

The estimated initial bubble size distribution is shown in Figure 5.

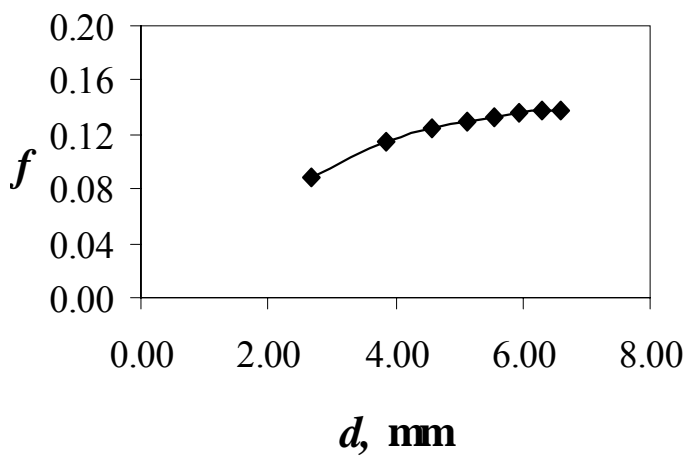

Fig 5. Estimated initial bubble size distribution for bubble column used by Prince and Blanch (1990).

The equilibrium size distribution estimated by the out lined model is compared with the experimental data obtained by Prince and Blanch ${ }^{10}$ at a distance $200 \mathrm{~cm}$ from the sparger. The result is shown in Figure 6 for velocity $5.2 \mathrm{~cm} / \mathrm{s}$.

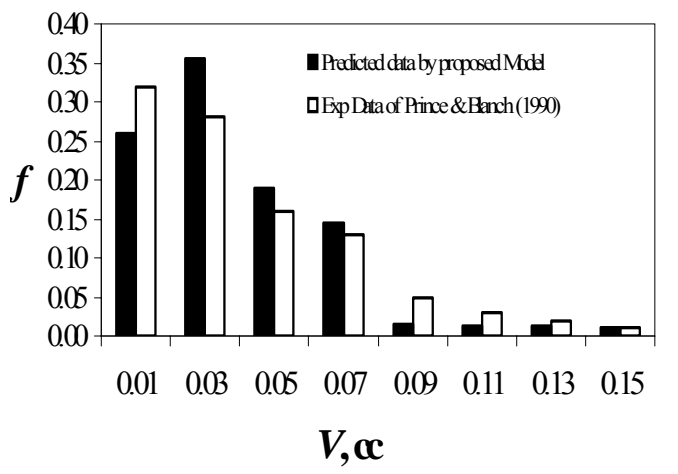

Fig 6. Comparison of the model with the experimental data of Prince and Blanch (1990) for velocity $5.2 \mathrm{~cm} / \mathrm{s}$.
Figures 3, 4 and 6 show satisfactory agreement between the experimental and predicted data, which validate the proposed model.

\section{Bubble size distribution}

When $d_{b}>d_{\max }$, the inertial force becomes greater than the surface force and bubble breaks up. On the other hand, bubbles whose diameters are smaller than $d_{\text {min }}$ are more prone to coalescence than the larger bubbles. There fore when bubbles are larger than $d_{\max }$ or smaller than $d_{\min }$ they are subject to break up or coalescence, respectively. Thus the equilibrium bubble size predicted by the proposed model would preferable lie between $d_{\min }$ and $d_{\max }$. However, from Figure 4, 5 and 7 it is evident that the bubble size distribution spread out beyond $d_{\max }$ and $d_{\min }$ for both predicted and experimental data. The reason behind such spreading out is the very low rate of bubble break up and coalescence beyond $d_{\min }$ to $d_{\max }$.

It is noted that the distribution frequencies of very small bubbles predicted by the model is less than that of the experimental ones. This is due to the fact that the break up may not be a simple binary split as assumed in the model. Prince et al. ${ }^{25}$ and others have reported that the bubble breakup is often accompanied by production of two primary bubbles and a number of smaller fragments. Incorporation of this effect would significantly alter the number of smaller bubbles predicted by the model. Furthermore, the complete range of eddy size is not considered in the model. The expression used for eddy number is only applicable for eddies within the inertial sub range. Eddies smaller than $20 \%$ of the bubble size was ignored. Such eddies may contribute to the breakage rate.

The model adopts a set of equations to predict the initial bubble size distribution. Those equations are based on the data for single orifice sparger and extended to the perforated plate sparger. This may cause error in initial bubble size determination, which in turn affects the equilibrium size distribution.

In present model the values of $d_{\max }$ and $d_{\min }$ are used as break-up and coalescence criteria. The equation for $d_{\min }$ has been established by a number of researchers. On the other hand, the expression for $d_{\max }$ is based on a single study. Further research is needed in this regard. The equation used for determining $U_{l, \max }$ is limited to Newtonian fluid and turbulent flow. In case of laminar flow or Non-Newtonian fluid different equations need to be applied. 


\section{Conclusion}

A model for determining equilibrium bubble size distribution in a bubble column has been developed. Both coalescence and breakup of bubbles have been considered in the model. The coalescence event was modeled by considering the collision rate of bubbles and the likelihood of collisions resulting in coalescence. Collisions due to turbulence, buoyancy and laminar shear were considered. The coalescence efficiency was determined by comparing the time required for coalescence and contact time of two bubbles in turbulent flow. Bubble breakup was modeled by examination of bubble interaction with turbulent eddies. Breakup was assumed to occur when bubbles encountered eddies of appropriate size and sufficient energy to cause rupture. In all cases the turbulence was assumed to be isotropic and the particles were considered to lie in the inertial subrange. The favorable comparison of reported data with the present model suggests that the model may be used to predict dispersed phase mixing rates in uncontaminated gas liquid systems. The model also provides a framework to optimize bubble column performance with regard to mass transfer rates.

\section{Notation}

$A=$ the Hamaker constant

$c=$ concentration of a surfactant species

$d_{b}=$ the bubble diameter, $\mathrm{m}$

$d_{\max }=$ the maximum stable bubble size, $\mathrm{m}$

$d_{\min }=$ the minimum stable bubble size, $\mathrm{m}$

$d_{g}=$ the geometric mean bubble size, $\mathrm{m}$.

$D=$ tank or column diameter, $\mathrm{m}$.

$E_{e}=$ the kinetic energy of the eddy, $\mathrm{Kg} . \mathrm{m}^{2} / \mathrm{s}^{2}$.

$E(k)=$ the energy of eddies of wave number $\mathrm{k}, \mathrm{kg}$. $\mathrm{m}^{3} / \mathrm{s}^{2}$.

$h=$ the film thickness between coalescing bubbles, $\mathrm{m}$.

$h_{o}=$ the initial film thickness, $\mathrm{m}$.

$h_{f}=$ the critical thickness where rupture occurs, $\mathrm{m}$.

$H=$ The un aerated liquid height, $\mathrm{m}$.

$k=$ The eddy wave number $\left(\mathrm{k}=1 / \mathrm{r}_{\mathrm{e}}, \mathrm{r}_{\mathrm{e}}\right.$ being the radius of the eddy), $\mathrm{m}^{-1}$.

$k_{e}=$ The wave number of the large energy containing eddies, $\mathrm{m}^{-1}$.

$k_{b}=$ The wave number of the corresponding bubble size, $\mathrm{m}^{-1}$.

$k_{d}=$ The wave number of the eddies of viscous dissipation, $\mathrm{m}^{-1}$.

$N(k)=$ the number of eddies of wave number $\mathrm{k}$ per mass of fluid and per unit interval of wave numbers.

$m=$ the eddy mass

$n_{i}, n_{j}=$ The concentration of bubbles of radius $\mathrm{r}_{\mathrm{bi}}, r_{b j}$

respectively, $\mathrm{m}^{-3}$.

$P_{1}=$ Pressure at the sparger, $\mathrm{N} / \mathrm{m}^{2}$.
$P_{2}=$ Absolute Pressure, $\mathrm{N} / \mathrm{m}^{2}$.

$P_{L M}=$ Log mean Pressure, $\mathrm{N} / \mathrm{m}^{2}$

$\mathrm{Q}=$ gas rate per orifice, $\mathrm{m}^{3} / \mathrm{s}$.

$Q_{M}=$ Mean gas rate, $\mathrm{m}^{3} / \mathrm{s}$.

$R=$ the radius of the column, $\mathrm{m}$.

$R_{d}=$ the radius of the liquid disk between the coalescing bubbles, $\mathrm{m}$.

$r_{e}=$ the eddy radius.

$S_{i j}=$ The collision cross sectional area of the bubbles, $\mathrm{m}^{2}$.

$t_{i j}=$ The time required for coalescence of bubbles of radius $\mathrm{r}_{\mathrm{bi}}$ and $\mathrm{r}_{\mathrm{bj}}$, sec.

$u_{e}=$ the eddy velocity, $\mathrm{m} / \mathrm{s}$

$u_{r}=$ The rise velocity of the particle, $\mathrm{m} / \mathrm{s}$.

$U_{l}=$ the liquid circulation velocity, $\mathrm{m} / \mathrm{s}$.

$U_{g}=$ The superficial gas velocity, $\mathrm{m} / \mathrm{s}$.

$U_{l, \max }=$ The velocity in the center of the column, $\mathrm{m} / \mathrm{s}$.

$x(E)=$ Energy distribution function

\section{Greek symbols}

$\varepsilon=$ The energy dissipation per unit mass, $\mathrm{m}^{2} / \mathrm{s}^{3}$.

$v=$ The kinematic viscosity, $\mathrm{m}^{2} / \mathrm{s}$.

$\rho_{c}=$ the liquid density, $\mathrm{Kg} / \mathrm{m}^{3}$.

$\rho_{f}=$ the fluid density, $\mathrm{Kg} / \mathrm{m}^{3}$.

$\sigma=$ The surface tension, $\mathrm{Kg} / \mathrm{s}^{2}$.

$\phi=$ Gas hold-up

$v_{t}=$ Turbulent Kinetic viscosity,

$\tau_{i j}=$ The contact times for the two bubbles of radius $\mathrm{r}_{\mathrm{bi}}$ and $\mathrm{r}_{\mathrm{bj}}$, sec.

$\mu_{c,} \mu_{\mathrm{d}}=$ the viscosities of the continuous and dispersed liquid phase respectively, $\mathrm{Kg} / \mathrm{m}$.s.

$\Gamma=$ gamma function.

$\theta^{\beta}=$ buoyancy driven collision rate, $\mathrm{m}^{-3} \cdot \mathrm{s}^{-1}$.

$\theta^{L S}=$ collision rate due to laminar shear, $\mathrm{m}^{-3} \cdot \mathrm{s}^{-1}$.

$\theta^{T}=$ collision rate due to Turbulence, $\mathrm{m}^{-3} \cdot \mathrm{s}^{-1}$.

\section{Subscripts}

$b=$ Bubble

$c=$ continuous phase

$d=$ dispersed phase

$e=$ eddy

$f=$ final

$g=$ gas

$i, j=$ particle $\mathrm{i}, \mathrm{j}$

$l=$ liquid

$T=$ total

\section{Superscripts}

- = Mean Value. 


\section{References}

1. Kolmogroff, A.N., Doklady Akad. Nauk. SSSR, $66: 825,1949$.

2. Hinze, J.O., Fundamentals of Hydrodynamic Mechanism of splitting in Dispersion Process, AIChE J., 1: 289, 1955.

3. Shinnar, R., Turbulence stabilized dispersions, Ph.D Thesis, Columbia University, 1957.

4. Valentas, K., Bilous, O., and Amundson, N. R. , Breakage and coalescence in dispersed phase systems, Ind. Eng. Chem, Fundam., 5: 533, 1966.

5. Valentas, K., Bilous, O., and Amundson, N. R. , Analysis of breakage in dispersed phase systems, Ind. Eng. Chem, Fundam., 5: 271, 1966.

6. Narsimhan, G., Gupta, J.P. and Ramkrishna, D. , A model for transitional breakage probability of droplets in agitated liquid- liquid dispersions, Chem Eng. Sci., 34:257, 1979.

7. Millies, M. and Mewes, D., Interfafacial area density in bubble flow, Chem. Eng-Proc. 38: 3073191999.

8. Lehr, F. and Mewes, D. A. Transport equation for the interfacial area density applied to bubble columns, Chem, eng. Sci. 56: 1159-1166, 2001.

9. Venneker, B., Derksen, J. and Van der Akker, H., Population balance modeling of aerated stirred vessels based on CFD, AIChE J, 48:673-685, 2002.

10. Prince, M. J., and Blanch, H. W., Bubble coalescence and Break up in air- sparged bubble columns, AIChE J, 36:1485-1499, 1990.

11. Colella, D., Vinci, D., Bagatin, R., Masi, M., and Bakr, E. A., A study on coalescence and breakage mechanisms in three different bubble columns, Chem. Eng. Sci., 4767-4777, 1999.

12. Akita, K. and Yoshida, F., Bubble size, interfacial area and liquid-phase mass transfer coefficient in Bubble columns, Ind. Eng. Chem., Process Des. Dev., 13(1):84-90, 1974.

13. Lage, P. L. C., The non-uniform bubble size distribution in bubble columns: the choice of bubble mean diameter for mass transfer, Proc. of XXVI ENEMP (Brazilian Congress on Particulate Systems) II: 631-636, 1996.

14. Hikita, H., Asai, S., Tanigawa, K., Segawa, K. and Kitao, M. , Gas hold-up in bubble columns, Chem. Eng. J., 20: 59-67, 1980.

15. Batchelor, G.K., The theory of homogeneous turbulence, Cambridge University Press, Cambridge 1970.

16. Friedlander, S. K., Dust, S., and Haze, A., Wiley, New York, 1977.

17. Walter, J., and Blanch, H. W., Liquid circulation patterns and their effect on gas hold up and axial mixing in bubble columns, Chem. Eng. Commun. , 19:243, 1983.

18. Coulaloglou, C. A., and Tavlarides, L.L., Description of interaction processes in agitated liquid-liquid dispersions, Chem. Eng. Sci, 32:1289, 1977.

19. Oolman, T. O., and Blanch, H. W., Bubble coalescence in air-sparged bioreactors, biotechnology and bioengineering, XXVIII, pp. 578-584,(1986).

20. Chesters, A. K., and Hofman G., Bubble coalescence in pure liquids, Appl. Scientif. Res., 38, 353, (1982).

21. Levich, V. G., Physicochemical hydrodynamics, Prentice-Hall, Englewood Cliffs, New Jersey, 1962.

22. Kennard, E. H., Kinetic theory of gases, McGrawHill, New York, 1938

23. Azbel, D., Two phase flows in chemical engineering, Cambridge University press, Cambridge 1981.

24. Angelidou, C., Psimopoulos, M. and Jameson, G. J., Size distribution functions of dispersions, Chem. Eng. Sci., 34: 671, 1979.

25. Prince, M., J. Walters, and H. W. Blanch, Bubble break-up in air sparged biochemical reactors, First Generation of Bioprocess Engineering, T K. Ghose, ed . 160, 1989 